\title{
TUNABLE IR FILTERS WITH INTEGRAL ELECTROMAGNETIC ACTUATORS
}

\author{
T.R. Ohnstein, J.D. Zook, and H.B. French \\ Honeywell Inc., Honeywell Technology Center \\ Plymouth, Minnesota 55441
}

\author{
H. Guckel, T. Earles, J. Klein, \\ and $P$. Mangat \\ University of Wisconsin Center for Applied \\ Microelectronics \\ Madison, Wisconsin 53706
}

\begin{abstract}
Tunable IR filters with integral electromagnetic drive actuators have been fabricated using the MEMS LIGA process at the University of Wisconsin. Two types of actuators with IR filter loads have been demonstrated: a single-pole solenoid-type actuator and a three-phase electromagnetic stepper motor drive. The IR filters were designed for a tunable range from 8 to $32 \mu \mathrm{m}$. The filter and actuator structures are fabricated from electroplated permalloy. Measurements of the magnetic properties of the permalloy show it to be a soft magnetic material with low coercive force $(0.3 \mathrm{Oe})$ and high permeability $(-65,000)$. An IR filter structure with a large displacement stepper motor has been demonstrated. The bi-directional stepper motor had a total travel of $1.7 \mathrm{~mm}$ with an average force per step of $2.4 \mathrm{mN}$ (basic step size of $25 \mu \mathrm{m}$ ) at a drive current of $45 \mathrm{~mA}$ per phase. This is the first demonstration of a MEMS linear electromagnetic stepping motor.
\end{abstract}

\section{INTRODUCTION}

Honeywell and the University of Wisconsin are developing microelectromechanical tunable IR filters for IR spectral analysis. The filters and integral actuators are fabricated by the MEMS LIGA process for making high-aspectratio metallic microstructures [1]. These devices will be the key elements in compact gas analyzers for applications in industrial process control and environmental monitoring. The tunable IR filter (Figure 1) is a mechanically deformable structure consisting of an array of parallel metal plates joined by spring flexures and driven by an integral linear actuator [2]. The plates form an array of identical waveguides whose transmittance properties can be varied by changing the spacing between them (Figures 2 and 3). The filter concept has been verified theoretically and demonstrated with IR filters fabricated with fixed dimension filter plate spacings $[2,3]$. The present paper describes the design, fabrication, and performance of the integral actuators used for tuning the filters, including the first working MEMS electromagnetic stepping motors (Figure 4).

\section{ACTUATOR DESIGN}

Tunable IR filters with two types of integral electromagnetic actuators have now been fabricated and demonstrated with tunable filter loads. Solenoid-type linear actuators, which have been used to drive spring-loaded microstructures and reported previously [1,2], have now been integrated with a tunable filter load. An electromagnetic stepper motor drive with an integral tunable filter has also been demonstrated with greater travel for filter tuning range and improved position control over the single-pole, solenoid-type actuator. The overall size of the filter and actuator structures ranges from $6 \times 6 \mathrm{~mm}$ to $6 \times 12 \mathrm{~mm}$ (Figure 4). The active area of the filters ranges from $1 \times 1 \mathrm{~mm}$ to $2.5 \times 2.5 \mathrm{~mm}$ (Figure 4). Filters have been fabricated with a wavelength cutoff range from

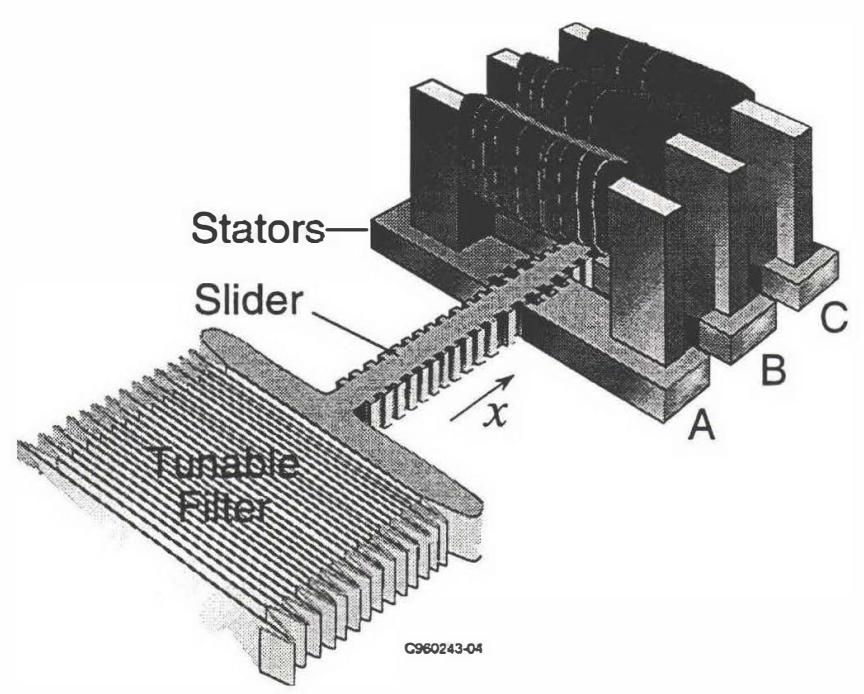

Figure 1. Tunable IR filter with integral stepping motor. The slider and tunable filter are electroplated from the same LIGA mask and assembled onto the stator and mounting posts fixed to the substrate. The electromagnets are assembled into the stators after the coils are wound.

8 to $32 \mu \mathrm{m}$. The filter structures with integral slider and spring supports are electro-plated onto a sacrificial layer, released, and assembled onto a substrate having magnetic pole faces and attachment posts (Figure 4). The mandrels for the electromagnets are plated separately, coils wound with copper magnet wire, and mounted with teeth that fit precisely into slots in the stators as shown in Figure 1. All the filter and actuator structures are fabricated using the MEMS LIGA process and are made of electroplated permalloy (approximately $78 \% \mathrm{Ni}, 22 \%$ $\mathrm{Fe})$.

The MEMS stepping motors are based on rotary switchedreluctance (SR) stepping motors that have well-known design principles $[4,5,6]$. In a linear motor, the rotor is replaced by a toothed slider that is coplanar with three identically toothed stators. In Figure 1, the teeth of stator $\mathrm{A}$ are aligned with the slider teeth, and stators B and C are displaced by one-third of a tooth period. The aligned position is the stable equilibrium position, meaning there is no force when $A$ is energized. This is also the maximum inductance position for coil $\mathrm{A}$. When the coil B current is also turned on, stator B pulls the slider to the right. When current $A$ is then turned off the slider teeth align with stator B. Thus sequential currents in coils A, B, and C drive the slider to the right by one tooth period for each drive cycle. Maximum slider displacement occurs when the magnetic holding force of one stator equals the restoring force of the support springs and filter load. 


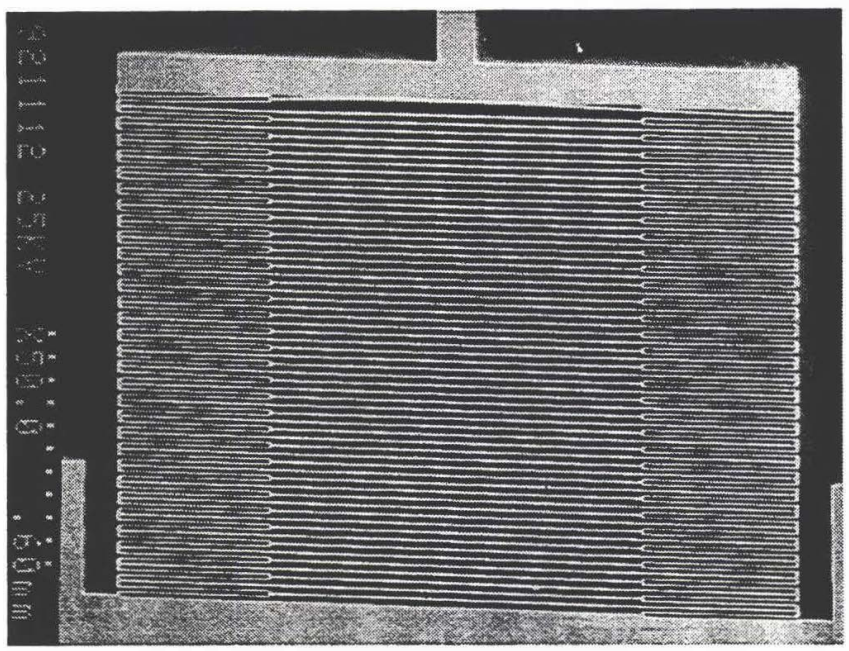

Figure 2. SEM photograph of tunable IR filter. The active filter area is in the center section with supporting spring flexures at each side. The active area is 1 by $1 \mathrm{~mm}$ (with filter in fully compressed position).

The stepper motor designs have $30-\mu \mathrm{m}$ teeth and $45-\mu \mathrm{m}$ spaces, with 10 teeth per stator and a basic step size of $25 \mu \mathrm{m}$ per phase. By energizing pairs of coils, the step size can be reduced to $12.5 \mu \mathrm{m}$.

The chosen tooth shape is based on large machines whose design is well established on the basis of modeling as well as empirical design rules [7]. The gaps between the slider and stator were nominally $2 \mu \mathrm{m}$, also scaled down from the large machines. Magnetic finite element modeling (FEM) was used to verify the baseline design and provide a basis of comparison with experiment. The two-dimensional model included two teeth from the stator, four from the slider, and a flux closure path. Magnetic fields were calculated for 16 different positions of the slider with respect to the stator, from the completely aligned case to the completely misaligned case. The force is equal to the change in stored magnetic field energy between positions, divided by the displacement.

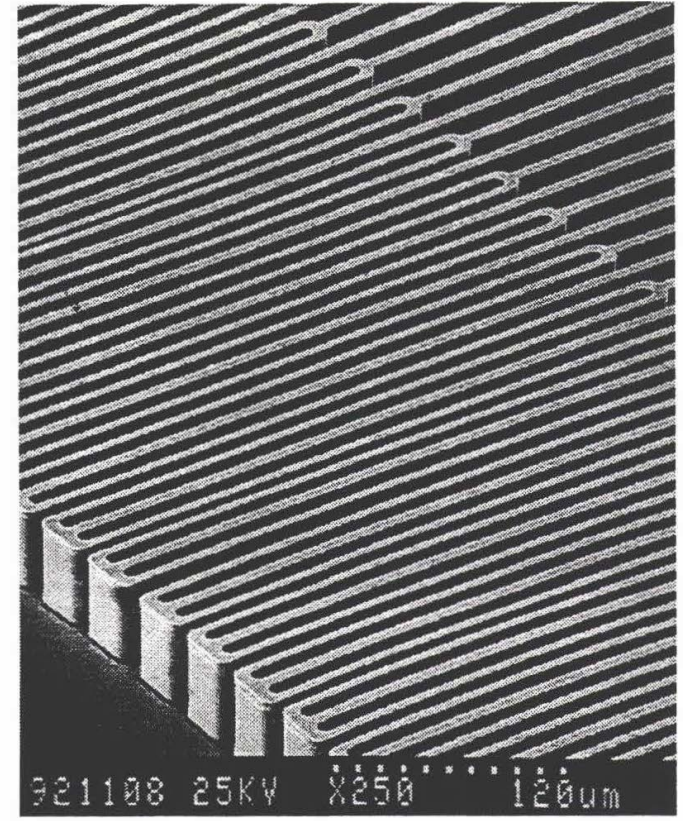

Figure 3. Enlarged view of filter spring flexures that join the filter plates at the sides of the active filter area.

In the baseline model for the stator and slider the nonlinear B-H relationship used was based on preliminary measurements of the permalloy properties. The B-H curve had a relative permeability of 2700 and a saturation magnetization $B_{S}$ of $1 \mathrm{~T}$.

Figure 5 shows the calculated force for six current (I) drive levels, from 1 to 32 ampere-turns in binary steps, for an electroplated permalloy thickness of $100 \mu \mathrm{m}$. The force is zero from symmetry considerations at both the aligned (stable) and misaligned (unstable) positions. For a linear material, each curve would have the same shape and an amplitude that scales as $I^{2}$.

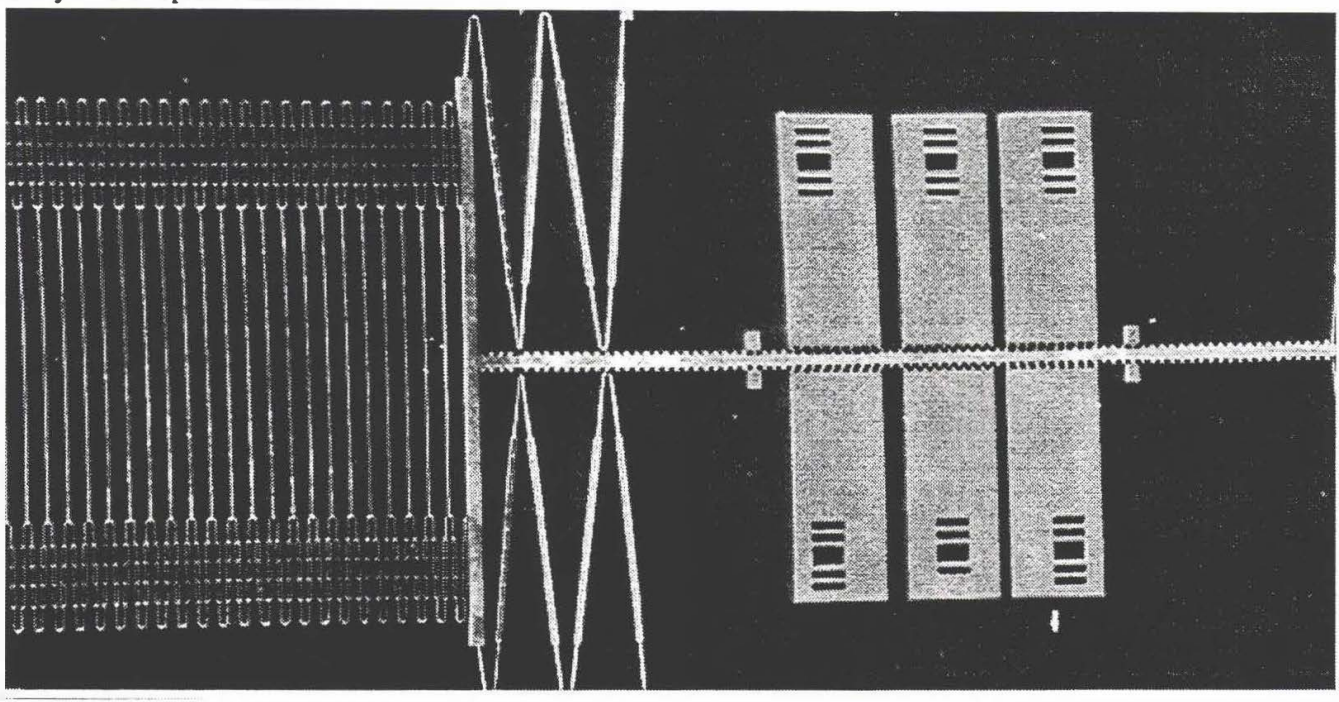

Figure 4. SEM photograph of an assembled IR tunable filter with a three-phase electromagnetic stepper motor drive. The filter, slider, and suspension spring structure is fabricated separately from the fixed substrate structures, mounting posts, and electromagnetic drive pole pieces. The drive coils (not shown) assembled in this photograph are mounted onto the pole pieces. 


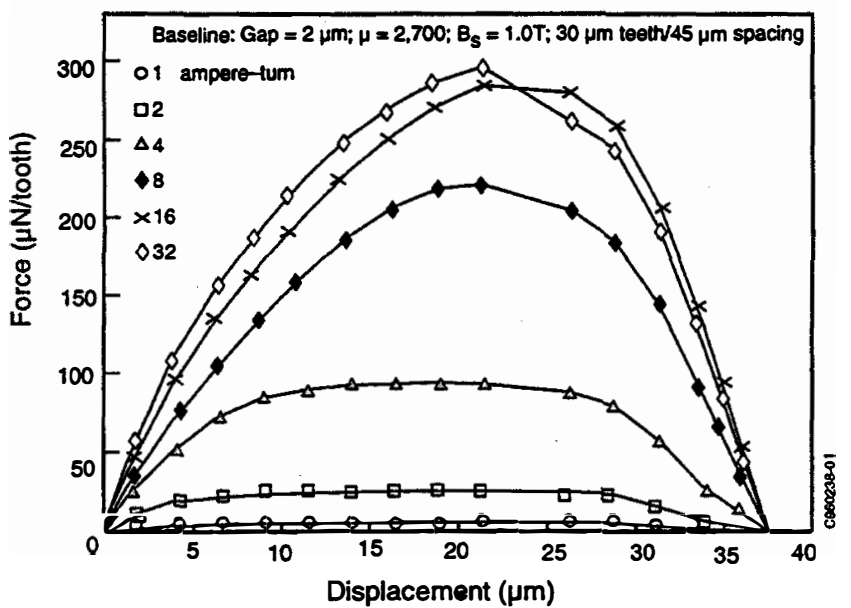

Figure 5. FEM calculation of the magnetic force as a function of slider position for six different current levels. The force is calculated from the change in magnetic field energy between two adjacent positions.

The actual curves change shape and bunch together with increasing current because of magnetic saturation. The maximum force that can be produced with this design and material parameters is about $300 \mu \mathrm{N} /$ tooth. The mean force at high drive levels is about $200 \mu \mathrm{N} /$ tooth or $4 \mathrm{mN} / \mathrm{stator}$ (for 20 teeth per stator).

Figure 6 illustrates the effects of changes in material properties and dimensions. The curves show the result of (1) increasing the gap to $5 \mu \mathrm{m}$ (to reduce material saturation effects); (2) increasing the material permeability to 15000 (either by annealing or composition changes); (3) increasing $B_{S}$ to 1.5 Teslas (by composition changes); and (4) using 45$\mu \mathrm{m}$-wide teeth separated by $30 \mu \mathrm{m}$ (for easier assembly). Figure 6 shows the calculated mean force per tooth between the aligned and misaligned positions plotted against the current drive level. Increasing the gap or the tooth width gives less force than the baseline case, while increasing the permeability has virtually no effect except at low drive levels (before material saturation sets in), and increasing $\mathrm{B}_{\mathrm{S}}$ increases the limiting force as $\mathrm{B}_{\mathrm{s}}{ }^{2}$. $B_{S}$ would increase with iron content in the alloy [8] at the risk of a tradeoff in mechanical properties of the structures with a change in the alloy.

\section{MATERIAL CHARACTERIZATION}

To measure the magnetic properties of the electroplated permalloy, toroid test structures (1.7 mm ID., $2.5 \mathrm{~mm} \mathrm{OD.)} \mathrm{were}$ fabricated along with the filter structures. The toroids were wound with a primary winding of 15 turns and a secondary winding of 30 turns, using 43-AWG wire. A known, timevarying current (proportional to $\mathrm{H}$ ) is applied to the primary, while measuring the voltage (proportional to $\mathrm{dB} / \mathrm{dt}$ ) across the secondary.

At present the primary is driven with a $10-\mathrm{Hz}$ triangular current waveform that extends into saturation. The very small cross-sectional area of the toroid produces a very small secondary voltage at this frequency, requiring a high-gain, lownoise, low-offset instrumentation amplifier. Integration of the voltage vs. time is done in software. The ascending and descending ramp waveforms are averaged before integrating, and

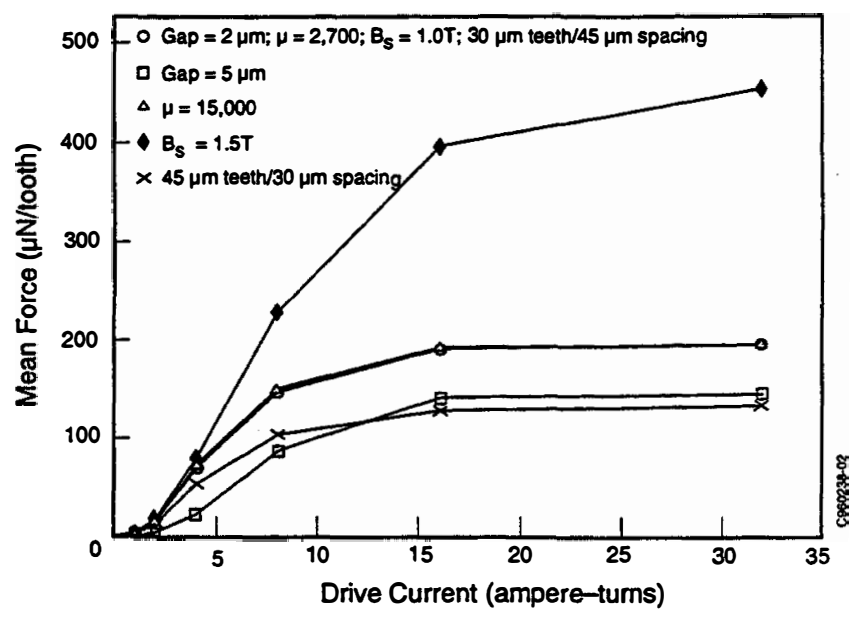

Figure 6. Calculated average force as a function of current for various changes in the assumptions of the model.

the offset voltage is chosen to make the slope of the B-H curve zero in saturation. The currents and voltages are converted to $\mathrm{H}$ and $\mathrm{B}$ using the physical dimensions of the toroid, the number of windings, the gain in the electronics, and the drive frequency. Using this procedure the B-H curve shown in Figure 7 was obtained for the permalloy toroid. A maximum permeability of about 65,000 , a saturation magnetization of about 9 kG (0.9 Teslas), and a coercivity of about 0.3 Oe were derived from this curve. These values are all of the same magnitude as, but somewhat poorer than, those expected for 78 Permalloy $(100,000,10.8 \mathrm{kG}$ and $0.05 \mathrm{Oe})[8]$.

The effective Young's modulus and mechanical fatigue properties of permalloy are being measured using the test structure shown in Figure 8 . It consists of a horseshoe electromagnet and a cantilever arm that is deflected toward the pole face when the drive coil is energized. The resonant frequency is easily measured with a gain/phase analyzer and is quite consistent from sample to sample at $20,100 \mathrm{~Hz}$, indicating a Young's modulus of $1.5 \times 10^{5} \mathrm{MPa}$, a little lower than the bulk value for permalloy. A circuit is being designed to drive the cantilever continuously to test the fatigue properties of the material as a function of strain amplitude. Indications to date are that electroplated permalloy is an excellent spring material [2].

\section{ACTUATOR PERFORMANCE}

Tunable IR filters with two types of integral electromagnetic actuators have now been fabricated: single-pole electromagnetic drives and three-phase electromagnetic stepper motors. The filter design in Figures 2 and 3 has been driven in resonance with a single-pole actuator with a total displacement of $250 \mu \mathrm{m}$. The three-phase stepper shown in Figure 4 has been driven the full amplitude $(1 \mathrm{~mm})$ in one direction. Displacement in the other direction has been limited so far to $0.7 \mathrm{~mm}$, apparently because of twisting of the flexures that cause them to hang up on the fixed parts. The twisting may be due to small variations in thickness of the permalloy springs.

Preliminary electrical measurements of the average actuator force during a step have been performed following the procedure used for large stepping and SR motors [9]. The inductance of an individual drive coil is measured as a function of dc drive current while the slider is held in a fixed position. 


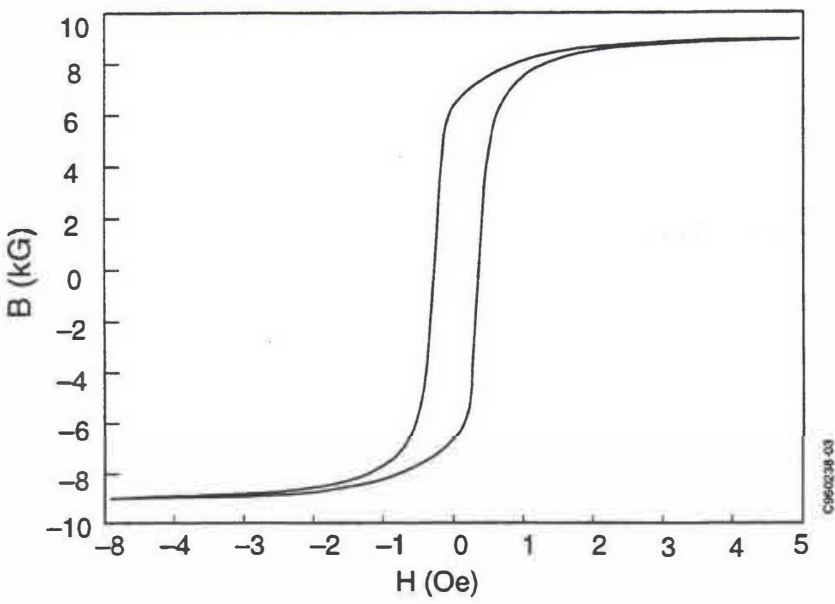

Figure 7. Measured B-H characteristics for an electroplated permalloy toroid. The average of many loops was taken and the voltage and current data were adjusted to make the curve symmetric.

The integral of the inductance with respect to current gives the flux linkage as a function of current. Integrating again with respect to current gives the energy stored in the magnetic field.

The measurements and integrations are performed for the slider in the aligned and unaligned positions for each individual drive coil. The difference in the two energies then gives the average drive force as a function of current (as shown in Figure 6 above) for the model. The procedure gave different force curves for each of the three coils, but the average force per step at the maximum test current of $45 \mathrm{~mA}$ was $2.4 \mathrm{mN}$. This value is in close agreement with the FEM analysis when the number of teeth and material thickness are considered. As fabrication imperfections in the stepping motor are eliminated, its output force will approach the design value of $4 \mathrm{mN} / \mathrm{step}$ and be more than adequate to drive the tunable filters, which are designed to have force constants of $1 \mathrm{mN} / \mathrm{mm}$ or less.

\section{CONCLUSIONS}

Tunable IR filter structures with integral electromagnetic drive actuators have been fabricated and demonstrated. A single pole, solenoid-type actuator and a three-phase electromagnetic stepper motor drive have been demonstrated with tunable IR filter loads. The IR filters were designed for a tunable range from 8 to $32 \mu \mathrm{m}$. The filter and actuator structures are fabricated with the MEMS LIGA process using electroplated permalloy. Measurements of the magnetic properties of the permalloy show it to be a soft magnetic material with low coercive force $(0.3 \mathrm{Oe})$ and high permeability ( 65,000). An IR filter with a large travel electromagnetic stepper motor drive was demonstrated. The bi-directional stepper motor had a total travel range of 1.7 $\mathrm{mm}$ with an average force per step of $2.4 \mathrm{mN}$ at a drive current of $45 \mathrm{~mA}$. A characterization of the transmission properties of the tunable IR filters has started as the next step in the development of the filters.

\section{ACKNOWLEDGMENTS}

This work was partially supported by ARPA Contract DABT63-93-C-0066. The motor drive electronics and demonstration were done by Ernie Satren. Material characterizations were done by Nick Demma. Additional

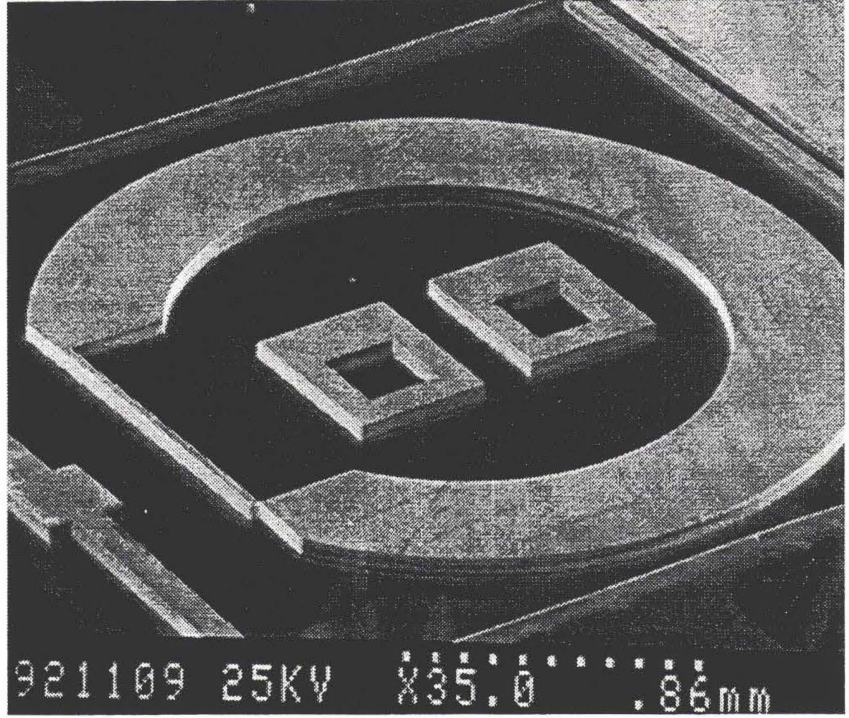

Figure 8. Photograph of unreleased electroplated parts including a structure for material characterization. The horseshoe magnet is wound with drive and pickup coils. The cantilever beam is driven in resonance. The beam is 1-mm long by 30- $\mu \mathrm{m}$ wide by $80-\mu \mathrm{m}$ tall. There is a $2-\mu \mathrm{m}$ gap between the end of the beam and the magnet.

processing of the structures was performed at Honeywell by Dawn Murphy.

\section{REFERENCES}

1. H. Guckel, et. al., "Electromagnetic Linear Actuators With Inductive Position Sensing For Micro Relay, Microvalve And Precision Positioning Applications," Technical Digest of Transducers '95, The 8th International Conference on SolidState Sensors and Actuators, Stockholm, Sweden, 6/25-29/95, pp. 324-327.

2. T. R. Ohnstein, et. al., "Tunable IR Filters Using Flexible Metallic Microstructures," Proc. 1995 Micro-ElectroMechanical Systems, Amsterdam, The Netherlands, 1/292/2/95, pp. $170-174$

3. J. A. Cox, et. al., "Optical Performance of High-Aspect LIGA Gratings," Proc. Micro-optics and Micromechanics Conf., SPIE Vol. 2383, San Jose. CA (Feb, 1995), pp. 17.

4. "Theory and Application of Step Motors," Ed. by B.C. Kuo, West Publishing Co., St. Paul (1974).

5. "Proc. Int. Conf. on Stepping Motors and Systems 19-20 Sept, 1979," Ed. by A. Hughes, University of Leeds (1980).

6. "Switched Reluctance Motor Drives-A reference book of collected papers," Ed. by T.J.E. Miller, Intertec Communications, Ventura, CA (1988).

7. T.J.E. Miller, "Switched Reluctance Drives," [6] above, pp.369, also M.R. Harris, et.al., "Limitations of Reluctance Torque in Doubly-Salient Structures," [6] above, pp 160-170.

8. D. Jiles, "Introduction to Magnetism and Magnetic Materials," Chapman and Hall, New York (1991).

9. G. Singh, "Mathematical modeling of step motors," [4] above, pp. 33-75. 